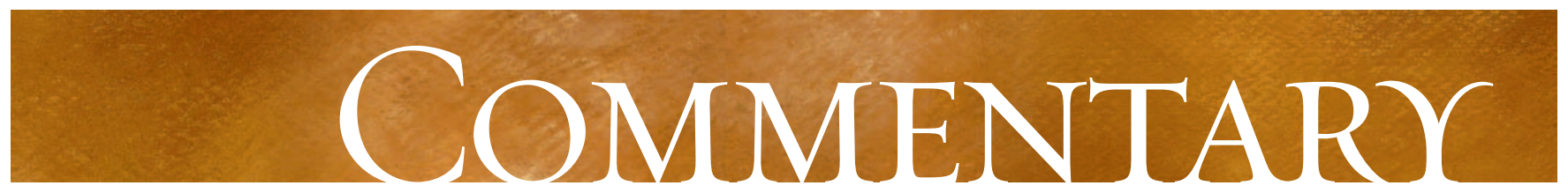

\title{
Auricular acupuncture for analgesia after arthroscopy
}

\section{Norman Buckley}

$\infty \quad$ See related article page I79

$\mathrm{A}$ cupuncture is entering the legislative mainstream of Canadian health care. The province of Ontario is about to legislate acupuncture as a therapeutic act under the jurisdiction of the Colleges responsible for the practitioner administering treatment (e.g., the College of Physicians and Surgeons of Ontario for physicians and the College of Nurses of Ontario for nurses). University courses in acupuncture are offered at both the University of Calgary and McMaster University. Colleges of traditional Chinese medicine exist in both British Columbia and Alberta. Traditional Chinese medicine is particularly noted for its focus on the maintenance of health, well-being and disease prevention. Acupuncture is extensively used in the treatment of chronic pain and in rehabilitation from musculoskeletal injuries. The moments when Western and traditional Chinese medicine intersect can be very interesting. The perioperative period may be one of these areas, when the analgesic effects of acupuncture can assist in recovery from Western-style surgery. As with any other aspect of medicine, a combination of clinical trials and anecdotal experience will be used to try to define the impact of and appropriate role for traditional Chinese medical techniques in clinical care.

Perioperative pain management is one area for the application of nonpharmacologic techniques, particularly if they may have good analgesic effect (promoting rapid discharge and recovery) with few of the side effects that complicate other analgesic treatments, especially opiates. Ineffective pain control, sedation, nausea and vomiting are common factors in delayed discharge or slow recovery after outpatient surgery. A simple, straightforward technique that offered good analgesia without sedation, nausea or vomiting would be widely embraced.

In this issue of CMAJ, Usichenko and colleagues ${ }^{1}$ present their experience with a trial of auricular acupuncture for analgesia after arthroscopy. Their article reveals the effectiveness of the technique, but also the limitations. The technique they examined in the study did provide marginally better analgesia t than the sham technique of invasive needle control: the meक्: dian dose of ibuprofen after discharge home was reduced by $400 \mathrm{mg}$. Most patients received at least some of the usual postoperative regimen of intravenous opiates (piritramide) titrated in the post-anesthesia care unit, followed by at-home use of ibuprofen and the weak opiate tramadol. The difference in postoperative use of analgesics between the treatment group (of whom $38 \%$ received no further analgesic) and the control group (19\%) was statistically significant, although $60 \%$ of those treated still required further analgesia. The numbers of patients who experienced nausea, vomiting and somnolence were similar between the 2 groups. It is unclear from the study whether the patients who were able to avoid other analgesics also avoided such adverse events. Finally, how to identify in advance those patients who could avoid other analgesics through use of auricular acupuncture is not stated. Because no costing was reported (the authors themselves identify cost-effectiveness as a potential topic for future study), we don't learn whether a set of acupuncture needles is more or less expensive than two 200-mg ibuprofen tablets. Since, in this study, separate acupuncturists were available to perform the procedure, educational costs to learn acupuncture, disposable costs (e.g., acupuncture needles v. analgesic tablets) and treatment costs for side effects (e.g., nausea) should be included in a future analysis.

\section{A technique offering good analgesia without sedation,}

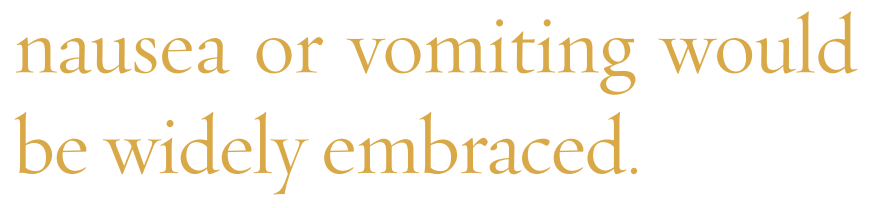

Certain acupuncture techniques clearly have powerful physiologic effects. Anecdotally, I have seen patients with severe peripheral vascular disease whose rest pain was relieved by acupuncture to the degree that they were able once again to exercise, and radial artery spasm relieved as effectively as by stellate ganglion block. Appiah and associates ${ }^{2}$ have reported successfully treating Raynaud's syndrome with acupuncture. Two of my colleagues have used acupuncture as the only anesthetic intervention for minor surgery (Drs. Angelica Fargas-Babjak, program chair, and Meena Nandagopal, faculty member, McMaster Contemporary Medical Acupuncture Education, McMaster University, Hamilton, Ont.: personal communication, 2005). Studies of anesthetized animals 
demonstrate acupuncture's ability to modulate gastric-acid secretion. ${ }^{3}$ Auricular acupuncture is shown here to have a measurable effect on analgesic needs, ${ }^{1}$ although it appears to be minor. To effectively integrate such a technique into perioperative care, one must be able to prospectively identify which patients the technique would permit to avoid other analgesics (and therefore their recovery-limiting side effects). The technique must also be readily teachable to the average anesthetist.

It seems to me that this is the sort of acupuncture research that needs to be done in the future: studies demonstrating the most effective use of its effect, not just illustrating an effect. That would be my challenge to the acupuncturists now: we believe that it works; just show us for whom. And then show us how to do it.

This article has been peer reviewed.
Norman Buckley is Associate Professor and Chair, Department of Anesthesia, Michael G. DeGroote School of Medicine, McMaster University, Hamilton, Ont.

Competing interests: None declared.

\section{REFERENCES}

I. Usichenko TI, Kuchling S, Witstruck T, et al. Auricular acupuncture for pain relief after ambulatory knee surgery: a randomized trial. CMAJ 2007;176(2):179-83.

2. Appiah R, Hiller S, Caspary L, et al. Treatment of primary Raynaud's syndrome with traditional Chinese acupuncture. JIntern Med I997;241:IIg-24.

3. Noguchi E, Hatyashi H. Increases in gastric acidity in response to electro-acupuncture stimulation of the hindlimb of anesthetized rats. Jpn J Physiol 1996;46:53-8.

Correspondence to: Dr. D. Norman Buckley, Department of Anesthesia, Michael G. DeGroote School of Medicine, McMaster University, 2UI-I200 Main St. W., Hamilton ON L8N 3Z5; fax go5 523-I224; buckleyn@mcmaster.ca
The complete picture

\section{on research.}

\section{PRACTICAL. RELEVANT. CMAJ IS NOW MORE COMPREHENSIVE THAN EVER.}

CMAJ's reputation and wide Canadian and international reach make it THE place to publish leading Canadian research. CMAJ publishes important peer-reviewed research within weeks of submission and ranks fifth among international general medical journals by impact factor, a measure of the scientific importance of a journal.

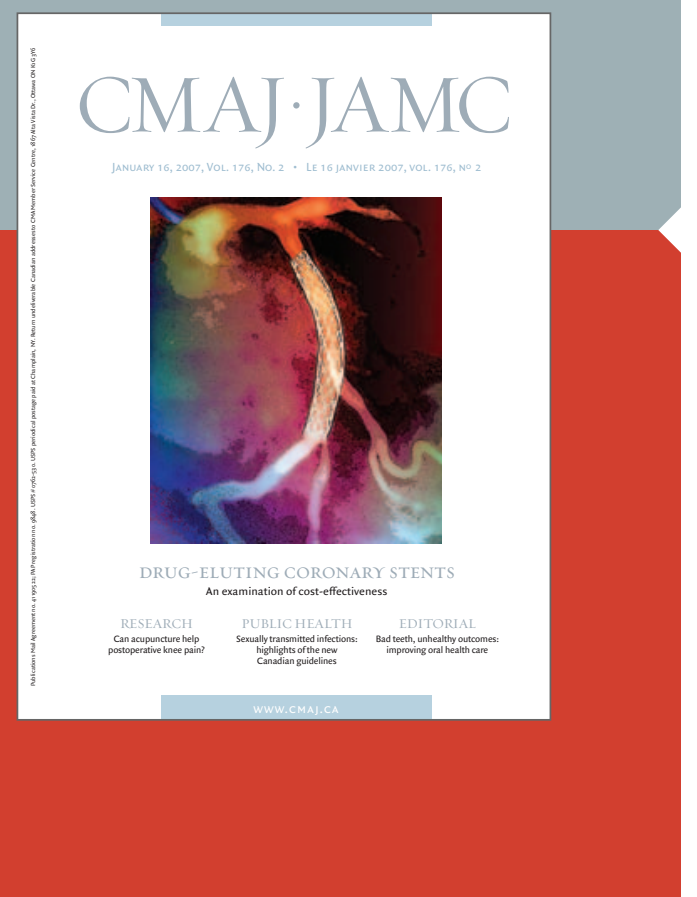

The essential read. ${ }^{\mathrm{TM}}$ 